\title{
AUTOMORPHISMS OF ONE-RELATOR GROUPS
}

\author{
Vladimir Shpilrain
}

\begin{abstract}
It is a well-known fact that every group $G$ has a presentation of the form $G=F / R$, where $F$ is a free group and $R$ the kernel of the natural epimorphism from $F$ onto $G$. Driven by the desire to obtain a similar presentation of the group of automorphisms $A u t(G)$, we can consider the subgroup $\operatorname{Stab}(R) \subseteq \operatorname{Aut}(F)$ of those automorphisms of $F$ that stabilize $R$, and try to figure out if the natural homomorphism $\operatorname{Stab}(R) \rightarrow \operatorname{Aut}(G)$ is onto, and if it is, to determine its kernel.

Both parts of this task are usually quite hard. The former part received considerable attention in the past, whereas the latter, more difficult, part (determining the kernel) seemed unapproachable. Here we approach this problem for a class of one-relator groups with a special kind of small cancellation condition. Then, we address a somewhat easier case of 2-generator (not necessarily one-relator) groups, and determine the kernel of the above mentioned homomorphism for a rather general class of those groups.
\end{abstract}

\section{Introduction}

Let $F=F_{n}$ be the free group of a finite rank $n \geq 2$ with a set $X=$ $\left\{x_{i}\right\}, 1 \leq i \leq n$, of free generators. Let $R$ be a normal subgroup of $F$, and $\operatorname{Stab}(R) \subseteq \operatorname{Aut}(F)$ the group of those automorphisms $\varphi$ of $F$ that stabilize $R$, i.e., $\varphi(R)=R$ (this does not necessarily mean that $\varphi$ fixes every element of $R)$.

Then there is the natural homomorphism $\rho: \operatorname{Stab}(R) \rightarrow \operatorname{Aut}(G)$, where $G=F / R$ (when we say the natural homomorphism, it means every element $s \in F$ maps onto the coset $s R$, and this extends to the mapping between groups of automorphisms). In some important cases, this homomorphism $\rho$ is known to be onto. This was established by Nielsen for surface groups; by Zieschang for somewhat more general one-relator and Fuchsian groups (see [14] and [15]), and, more recently, a number of results in this direction have been published; we only mention a couple of them which are in line with the subject of the present paper. Lustig, Moriah and Rosenberger [7] completely characterized Fuchsian groups with the property above. Bachmuth, Formanek

1991 Mathematics Subject Classification: Primary 20F28, secondary 20E36, 57M05. 
and Mochizuki [1] established this property for a class of 2-generator groups. On the other hand, it is known by now that for many groups $G$, the homomorphism $\rho$ is not onto - see [6] or [12] for a survey.

When $\rho: \operatorname{Stab}(R) \rightarrow \operatorname{Aut}(G)$ happens to be onto, it is natural to try to determine its kernel, since that would give a presentation of the group $\operatorname{Aut}(G)$ as $\operatorname{Stab}(R) / \operatorname{Ker}(\rho)$, and this, in turn, could give a presentation of $\operatorname{Aut}(G)$ by generators and defining relations (at least in the case where $G$ is finitely presented) if one uses results of McCool [9] on presentation of $\operatorname{Stab}(R)$.

It is clear that the group $\operatorname{Inn}_{R}$ of inner automorphisms of $F$ induced by elements of $R$, is always contained in $\operatorname{Ker}(\rho)$. In many cases, this is the whole $\operatorname{Ker}(\rho)$ (see Theorem 1.1 below). However, in several important situations $\operatorname{Ker}(\rho)$ happens to be bigger than $\operatorname{Inn}_{R}$. This is the case, in particular, with surface groups:

Example 1.(a) Let $r=x_{1}^{2} x_{2}^{2} \ldots x_{n}^{2}$, and $\varphi: x_{1} \rightarrow x_{1} \cdot r^{-x_{1}^{-2}}=x_{1}^{-1} \cdot x_{n}^{-2}$. $\ldots \cdot x_{2}^{-2} ; x_{i} \rightarrow x_{i}, i \neq 1$ (our notation is $x^{y}=y x y^{-1}$ ). Then $\varphi$ is obviously a non-inner automorphism of the free group $F_{n}$, but it induces the identical automorphism of the group $F_{n} / R$.

(b) A similar automorphism works in the orientable case, where $r=\left[x_{1}, x_{2}\right]$. $\left[x_{3}, x_{4}\right] \cdot \ldots \cdot\left[x_{2 m-1}, x_{2 m}\right]$ (we assume $n=2 m \geq 4$, and our commutator notation is $\left.[x, y]=x^{-1} y^{-1} x y\right)$ :

$\varphi_{o}: x_{1} \rightarrow x_{1} \cdot r=x_{2}^{-1} x_{1} x_{2} \cdot\left[x_{3}, x_{4}\right] \cdot \ldots \cdot\left[x_{2 m-1}, x_{2 m}\right] ; x_{i} \rightarrow x_{i}, i \neq 1$.

Example 2.(a) A little more sophisticated example is provided (in the nonorientable case) by the following automorphism (here $r=x_{1}^{2} x_{2}^{2} \ldots x_{n}^{2}$ ): $\psi: x_{1} \rightarrow r^{-x_{1}^{-2}} \cdot x_{1} \cdot r^{-x_{n}^{-2}} \cdot r_{1}^{x_{1}^{-2}} ; x_{i} \rightarrow x_{i}, i \neq 1, n ; x_{n} \rightarrow r^{-x_{1}^{-2}} \cdot x_{n} \cdot r^{x_{1}^{-2}}$.

(b) Based on a similar idea, we get the following automorphism in the orientable case:

$\psi_{o}: x_{1} \rightarrow r^{\left[x_{2}, x_{1}\right]} \cdot x_{1} ; x_{2} \rightarrow x_{2}^{r^{\left[x_{2}, x_{1}\right]}} ; x_{i} \rightarrow x_{i}, i \neq 1,2$.

It is not quite obvious that the automorphisms from Examples 1 and 2 belong to $\operatorname{Stab}(R)$; it is however obvious that we have $\varphi(R) \subseteq R$ for any of them; now $\varphi(R)=R$ follows from the fact that surface groups are hopfian (this was established by Hopf himself).

These are not the only examples of non-inner automorphisms of a free group $F$ that induce the identical automorphism of the corresponding surface group $F / R$. In fact, the group $\operatorname{Ker}(\rho) / \operatorname{Inn}_{R}$ appears to be rather big; at least, it is non-periodic and non-abelian (the automorphisms $\varphi$ and $\psi$ from Examples 1(a) and 2(a) do not commute modulo $\operatorname{Inn}_{R}$ ). The problem of an actual description of the group $\operatorname{Ker}(\rho)$ for any of the surface groups (of rank $>2$ ) 
remains open (to the best of my knowledge). We note that Dicks and Formanek [5] recently described (in particular) the kernel of a natural homomorphism ("collapse") from the automorphism group of the fundamental group of an orientable surface with one puncture onto the corresponding (standard) surface group.

Here we suggest a possible approach to the problem of describing $\operatorname{Ker}(\rho)$, which works for many one-relator groups that satisfy a strong type of small cancellation condition, in particular for groups that are in some sense close to surface groups. But, as it happens also in some other instances, the situation with surface groups appears to be on the border between difficult and impossible, so it is not clear at the moment if this approach can be pushed through for surface groups as well.

Before we give the statement of our first result, we need to say a couple of words about the Whitehead graph $W h(u)$ of a free group element $u$. The vertices of this graph correspond to the elements of the generating set $X$ and their inverses. If the word $u$ has a subword $x_{i} x_{j}$, then there is an edge in $W h(u)$ that connects the vertex $x_{i}$ to the vertex $x_{j}^{-1}$; if $u$ has a subword $x_{i} x_{j}^{-1}$, then there is an edge that connects $x_{i}$ to $x_{j}$, etc. We note that usually, there is one more edge (the external edge) included in the definition of the Whitehead graph: this is the edge that connects the vertex corresponding to the last letter of $u$, to the vertex corresponding to the inverse of the first letter. We shall not include the external edge in $W h(u)$; instead, we shall consider the Whitehead graph $W h(\bar{u})$ of a cyclic word $\bar{u}$ when necessary, in which case, of course, the external edge is included.

Theorem 1.1. Let $G=F_{n} / R, n \geq 3$, be a one-relator group with a relator $r$ and with the following property: $G$ satisfies a small cancellation condition $C^{\prime}(\lambda), \lambda \leq 1 / 6$, and the Whitehead graph of any subword of length $\geq(1-$ $3 \lambda)|r|$ of the word $r$ or any of its cyclic permutations, is 2-connected (i.e., is connected and does not have a cut vertex). Let $\rho: \operatorname{Stab}(R) \rightarrow \operatorname{Aut}(G)$ be the natural homomorphism. Then $\operatorname{Ker}(\rho)=\operatorname{Inn}_{R}$.

If $n=2$, there might be some additional automorphisms in $\operatorname{Ker}(\rho)$, namely, inner automorphisms induced by elements $u \in F$ such that $u R$ is central in $G=F / R$. We treat 2-generator groups separately, in Theorem 1.3.

We note that it is easy and straightforward to check if a given one-relator group satisfies the conditions of Theorem 1.1.

Although surface groups do not satisfy all those conditions, there are "similar" groups that do; those are, for example, one-relator groups with the relator of the form $\left(x_{1}^{2} x_{2}^{2} \ldots x_{n}^{2}\right)^{p}, p \geq 2$, or $\left(\left[x_{1}, x_{2}\right] \cdot\left[x_{3}, x_{4}\right] \cdot \ldots \cdot\left[x_{2 m-1}, x_{2 m}\right]\right)^{p}, p \geq 2$. 
(In general, one-relator groups with a "very long" relator tend to satisfy those conditions).

Note also that the stabilizer of a (cyclic) word $\left(x_{1}^{2} x_{2}^{2} \ldots x_{n}^{2}\right)^{p}$, or $\left(\left[x_{1}, x_{2}\right]\right.$. $\left.\left[x_{3}, x_{4}\right] \cdot \ldots \cdot\left[x_{2 m-1}, x_{2 m}\right]\right)^{p}$ is the same as that of $x_{1}^{2} x_{2}^{2} \ldots x_{n}^{2}$, or $\left[x_{1}, x_{2}\right] \cdot\left[x_{3}, x_{4}\right] \cdot \ldots$. $\left[x_{2 m-1}, x_{2 m}\right]$, respectively, and that the homomorphism $\rho: \operatorname{Stab}(R) \rightarrow \operatorname{Aut}(G)$ is onto for any of the corresponding one-relator groups $G=F / R$ (see [11] or [12]). Therefore, we have:

Corollary 1.2. Let $G=F_{n} / R, n \geq 3$, be a one-relator group with the relator of the form $\left(x_{1}^{2} x_{2}^{2} \ldots x_{n}^{2}\right)^{p}, p \geq 2$, or $\left(\left[x_{1}, x_{2}\right] \cdot\left[x_{3}, x_{4}\right] \cdot \ldots \cdot\left[x_{2 m-1}, x_{2 m}\right]\right)^{p}, p \geq 2$. Then $\operatorname{Aut}(G)=\operatorname{Stab}(R) / \operatorname{Inn}_{R}$.

Theorem 1.1 is proved the following way: first we apply small cancellation theory to make sure that we have a sufficiently large fragment of $r^{ \pm 1}$ (or some of its conjugates) in every element of $R$, and then use this large fragment to show that the Whitehead graph of a cyclically reduced element of the form $x_{i} \cdot s, s \in R$, cannot be the Whitehead graph of a primitive element of a free group, since those large fragments appear to be "primitivity-blocking" because the Whitehead graph of such a fragment does not have a cut vertex. This latter observation is essentially due to E.Turner (informal communication).

One more remark about automorphisms of surface groups is in order. The group of outer automorphisms of a surface group is known to be isomorphic to the mapping class group of the corresponding surface, and this latter group was studied extensively by a number of people. In particular, Lickorish, Birman and others obtained several different presentations of mapping class groups based on various geometric ideas (see [3]). Recently, a very "economical" presentation was found by Wajnryb 13. These presentations however do not help much in obtaining a presentation of the whole group of automorphisms of a surface group.

Finally, we consider the problem of determining $\operatorname{Ker}(\rho)$ for 2-generator (not necessarily one-relator) groups. These groups are easier to handle because of a very convenient criterion of primitivity for an element of the free group of rank 2, which can be found in [4] and (somewhat disguised) in [10]. Based on this criterion, we prove:

Theorem 1.3. Let $R \subseteq\left[F_{2}, F_{2}\right]$. If $\varphi \in \operatorname{Ker}(\rho)$ (where, as before, $\rho$ : $\operatorname{Stab}(R) \rightarrow \operatorname{Aut}(G)$ is the natural homomorphism), then $\varphi$ is an inner automorphism induced by an element $s \in F_{2}$ such that $s R$ is central in $F_{2} / R$.

In particular, if a group $G=F_{2} / R$ has trivial centre, then $\operatorname{Ker}(\rho)=\operatorname{In} n_{R}$. 
We emphasize once again that when the rank of a group $G$ is bigger than 2 , the situation becomes much more complicated. In particular, it is not known what $\operatorname{Ker}(\rho)$ is when $G$ is a free metabelian group of rank $>2$. It is not even known whether or not $\operatorname{Ker}(\rho)$ is finitely generated as a normal subgroup of $\operatorname{Aut}(F)=\operatorname{Stab}(R)$ in that case. What is known is that $\rho$ is onto for a free metabelian group of rank $>3$ (see [2]). We draw a special attention to free metabelian groups here because the problem of determining $\operatorname{Ker}(\rho)$ for those groups is closely related to a notorious problem of combinatorial group theory and algebraic topology - to the problem of the Gassner representation of a pure braid group being faithful (see [3], Section 3.3).

\section{One-relator groups}

Proof of Theorem 1.1. We start by recalling a well-known property of the Whitehead graph of a free group element. If an element is primitive and cyclically reduced, then its Whitehead graph has a cut vertex, i.e., a vertex that, having been removed from the graph together with all incident edges, increases the number of connected components of the graph.

Therefore, if we want to prove that some element of a free group is not primitive, it is sufficient to show that this element has a subword whose Whitehead graph is 2-connected.

Now, by way of contradiction, suppose there is an automorphism $\varphi$ of the free group $F_{n}$ that takes $x_{i}$ to $x_{i} \cdot s_{i}, 1 \leq i \leq n$, where $s_{i} \in R$, and at least one of the $s_{i}$, say, $s_{1}$, is non-trivial.

We need to have some of the elements $x_{i} \cdot s_{i}$ cyclically reduced to apply the property of the Whitehead graph discussed above. Suppose, for example, that $u_{1}=x_{1} \cdot s_{1}$ is cyclically reduced (this means, in particular, that there is no cancellation between $x_{1}$ and $s_{1}$ ). Then, $u_{1}$ being primitive, the Whitehead graph $W h\left(u_{1}\right)$ must have a cut vertex. However, there is a subword of $u_{1}$ whose Whitehead graph has no cut vertex. Indeed, a small cancellation condition $C^{\prime}(\lambda), \lambda \leq 1 / 6$ implies that every element of $R$ has a subword whose length is more than $(1-3 \lambda)|r|$ and which is a subword of some cyclic permutation of $r^{ \pm 1}$ - see [8], Theorem V.4.4. Now the conditions of Theorem 1.1 imply that the Whitehead graph of such a subword has no cut vertex, and therefore neither does $W h\left(u_{1}\right)$, hence a contradiction.

If there is a cancellation between $x_{1}$ and $s_{1}$, i.e., if $s_{1}$ is of the form $x_{1}^{-1} s_{1}^{\prime}$ (but not of the form $s_{1}^{\prime} x_{1}^{-1}$ ), then we might lose one letter in our long subword described in the previous paragraph; that is why we require the condition on the Whitehead graph to be satisfied by subwords of length $\geq(1-3 \lambda)|r|$, not 
just $>(1-3 \lambda)|r|$ as it appears in Theorem V.4.4 of [8].

Suppose now that all of $x_{i} \cdot s_{i}$ are cyclically reducible; that means, in particular, that there might be cancellations between $x_{i}$ and $s_{i}$. Then start composing our automorphism $\varphi$ with automorphisms $\psi_{i, k}$, where $k$ runs through integers, $2 \leq i \leq n$, and $\psi_{i, k}$ takes $x_{1}$ to $x_{1} x_{i}^{k}$ and fixes other generators. Thus, $\psi_{i, k} \circ \varphi$ takes $x_{1}$ to $v_{1}=x_{1} \cdot s_{1} \cdot\left(x_{i} \cdot s_{i}\right)^{k}$. If for some $i, k$ the element $v_{1}$ is cyclically reduced (of course, it is still primitive), then we are done since the previous argument applies.

If for all pairs $(i, k)$ the element $v_{1}$ is not cyclically reduced, this can only mean that every $\varphi\left(x_{i}\right)$ has a form $w_{i}^{g}=\left(x_{i} \cdot y_{i}\right)^{g}$ for some $g \in F, y_{i} \in R$. In that case, we see that the element $x_{i} \cdot y_{i}$ is itself primitive (as a conjugate of a primitive element), but we have seen that this is only possible if $y_{i}=1$, i.e., if our automorphism $\varphi$ was just the conjugation by $g$.

Thus, we have shown so far that only inner automorphisms of a free group might belong to $\operatorname{Ker}(\rho)$. Since every $n$-generator one-relator group has trivial centre provided $n \geq 3$ (see [8], Proposition II.5.22), this implies $\operatorname{Ker}(\rho)=\operatorname{Inn}_{R}$, and this completes the proof of Theorem 1.1.

\section{Two-generator groups}

Proof of Theorem 1.3. We start by recalling a convenient necessary condition of primitivity in $F_{2}$ (see [4]):

- if $w$ is a primitive element of $F_{2}$, then some conjugate of $w$ can be written in the form $x_{1}^{k_{1}} x_{2}^{l_{1}} \ldots x_{1}^{k_{m}} x_{2}^{l_{m}}$, so that some of $x_{i}$ occurs either solely with exponent 1 or solely with exponent -1 .

To prove Theorem 1.3, it is sufficient to prove the following statement: if a cyclically reduced primitive element of $F_{2}$ has a form $x_{1} \cdot c, c \in\left[F_{2}, F_{2}\right]$, then $c=1$. Indeed, if we prove it, it will follow that the only situation where an automorphism of $F_{2}$ may induce the identical automorphism of $G$, is where both generators $x_{1}$ and $x_{2}$ are taken to their conjugates. In that case, by a well-known result of Nielsen (see e.g. [8], Proposition I.4.5), this automorphism must be inner. The result follows.

Now we prove the statement. If $c \in\left[F_{2}, F_{2}\right]$, then both $x_{1}$ and $x_{2}$ occur in $c$ both with positive and negative exponents. Therefore, the only way for an element of the form $x_{1} \cdot c$ to be primitive is to have a cancellation of $x_{1}$ with the first letter of $c$, which has to be $x_{1}^{-1}$. Thus, let $c=x_{1}^{-1} \cdot c_{1}$, where $c_{1}$ has only positive occurences of $x_{1}$. But this is possible only if $c_{1}=x_{2}^{k} x_{1} x_{2}^{-k}$; in that case, we have $x_{1} \cdot c=x_{2}^{k} x_{1} x_{2}^{-k}$, a cyclically reducible element, contrary 
to our assumption. This completes the proof.

\section{Acknowledgement}

I am grateful to J.McCool for useful discussions.

\section{References}

[1] S.Bachmuth, E.Formanek, H.Y.Mochizuki, IA-automorphism of certain two-generator torsion-free groups, J. Algebra 40 (1976), 19-30.

[2] S.Bachmuth, H.Y.Mochizuki, $\operatorname{Aut}(F) \rightarrow \operatorname{Aut}\left(F / F^{\prime \prime}\right)$ is surjective for free group $F$ of rank $\geq 4$, Trans. Amer. Math. Soc. 292 (1985), 81101.

[3] J.S.Birman, Braids, links and mapping class groups, Ann. Math. Studies 82, Princeton Univ. Press, 1974.

[4] M.Cohen, W.Metzler and B.Zimmermann, What does a basis of $F[a, b]$ look like?, Math. Ann. 257 (1981), 435-445.

[5] W.Dicks, E.Formanek, Automorphism subgroups of finite index in algebraic mapping class groups, J. Algebra 189 (1997), 58-89.

[6] C.K.Gupta, V.Shpilrain, Lifting automorphisms: a survey, Groups '93 Galway/St. Andrews, Vol. 1 (Galway, 1993), 249-263, London Math. Soc. Lecture Note Ser. 211, Cambridge Univ. Press, Cambridge, 1995.

[7] M.Lustig, Y.Moriah, G.Rosenberger, Automorphisms of Fuchsian groups and their lifts to free groups, Canad. J. Math. 41 (1989), 123131.

[8] R.Lyndon, P.Schupp, Combinatorial Group Theory, Series of Modern Studies in Math. 89. Springer-Verlag, 1977.

[9] J.McCool, Some finitely presented subgroups of the automorphism group of a free group, J. Algebra 35 (1975), 205-213.

[10] R.P.Osborne, H.Zieschang, Primitives in the free group on two generators, Invent. Math. 63 (1981), 17-24.

[11] G.Rosenberger, Alternierende Produkte in freien Gruppen, Pacific J. Math. 78 (1978), 243-250. 
[12] G.Rosenberger, Applications of Nielsen's reduction method to the solution of combinatorial problems in group theory: a survey, Homological group theory (Proc. Sympos., Durham, 1977), pp. 339-358, London Math. Soc. Lecture Note Ser. 36, Cambridge Univ. Press, CambridgeNew York, 1979.

[13] B.Wajnryb, Mapping class group of a surface is generated by two elements, Topology 35 (1996), 377-383.

[14] H.Zieschang, Über Automorphismen ebener diskontinuierlicher Gruppen, Math. Ann. 166 (1966), 148-167.

[15] H.Zieschang, E.Vogt, H.-D.Coldewey, Surfaces and planar discontinuous groups, Translated from the German by John Stillwell. Lecture Notes in Mathematics, 835. Springer, Berlin, 1980.

Department of Mathematics, The City College of New York, New York, NY 10031

e-mail address: shpil@groups.sci.ccny.cuny.edu 